Relations industrielles

Industrial Relations

\title{
Labour Costs in European Industry, International Labour Office, Geneva, 1959, 170 pp. \$1.25
}

\section{Gérard Dion}

Volume 14, numéro 4, octobre 1959

URI : https://id.erudit.org/iderudit/1022145ar

DOI : https://doi.org/10.7202/1022145ar

Aller au sommaire du numéro

Éditeur(s)

Département des relations industrielles de l'Université Laval

ISSN

0034-379X (imprimé)

1703-8138 (numérique)

Découvrir la revue

Citer ce compte rendu

Dion, G. (1959). Compte rendu de [Labour Costs in European Industry, International Labour Office, Geneva, 1959, 170 pp. \$1.25]. Relations industrielles / Industrial Relations, 14(4), 624-625.

https://doi.org/10.7202/1022145ar

Tous droits réservés (C Département des relations industrielles de l’Université Laval, 1959
Ce document est protégé par la loi sur le droit d'auteur. L’utilisation des services d'Érudit (y compris la reproduction) est assujettie à sa politique d'utilisation que vous pouvez consulter en ligne.

https://apropos.erudit.org/fr/usagers/politique-dutilisation/ 
vrages qu'il a utilisés et l'autre, les ouvrages dont il suggère la lecture. Un index bien fait permet aussi une consultation rapide.

Tout au cours de son exposé l'auteur illustre ses allégations de multiples faits tirés de l'expérience. Il ne se gêne pas pour exprimer son opinion, donner des noms. Les chapîtres concernant la corruption et le communisme sont particulièrement caractéristiques.

« Racketeering is a form of union pathology. Like Communist unionism, discussed in the next chapter, racketeering is the introduction of foreign bodies into the content of unionism. Inherent in free unions is that they are self-governing entities designed to improve the conditions under which their members work... When racketeering or Communist influences gain a foothold, the union is wrenched from its moorings and its purposes and government are deliberately distorted to serve the cupidity of outsiders or insiders for naked poser of money or both. Racketeering and Communist domination in unions are necessarily a tampering with the essential integrity of the union as we know union in a free society. So, in a sense, a racketeering or Communist-dominated union is a contradiction in terms. It can't really be a union if it is controlled by racketeers and Communists. 》

Il a bien résumé l'autonomisme que veut garder le mouvement ouvrier américain par rapport aux autres organisations extérieures:

« Underlying the idea of a labor movement as it has developed in the United States is the fundamental principle that the movement cannot properly sumit to domination by organized political, religious, governmental, and employer power groupes. External domination of this kind is regarded as incompatible with a free labor movement - or with a free union. Only political domination has been a serious problem in the United States, but that issue was settled for the last time with the ouster of the Communist-controlled unions from the family of unions. This view of the nature of a free moviement distinguishes the labor movement in the United States from many labor movements in the rest of the world. \$
Malgré ses liens avec le mouvement ouvrier, l'auteur a cherché à être objectif et à présenter le syndicalisme tel qu'il le voit avec ses faiblesses, ses difficultés. Ce livre est un témoignage, un témoignage de première main qui nous montre le mouvement ouvrier tel que le considèrent eux-mêmes les travailleurs. C'est ce qui fait sa valeur. On ne peut manquer d'y recourir si l'on veut porter un jugement éclairé sur ce phénomène si important dans notre vie économique et sociale.

\section{GÉRARD DION}

\section{Labour Costs in European Industry,} International Labour Office, Geneva, 1959,170 pp. $\$ 1.25$

Lors de le première conférence régionale européenne de l'Organisation internationale du travail tenue à Genève au début de 1955 , il a été question de coopération économique et on a longuement discuté de différences qui existent entre les pays dans les salaires et les coûts du travail. Il a été recommandé qu'une enquête soit entreprise sur ce sujet. Cet ouvrage en est le résultat.

Les compléments aux salaires ont pris une importance considérable en ces dernières années. Il est évident qu'aujourd'hui des statistiques sur les salaires sont inadéquates tant pour indiquer le coût de la main d'oeuvre que le revenu des travailleurs. \& L'objet de cette étude est, entre autres, d'offrir une mesure statistique objective des différences dans le coût de la main d'oeuvre par heure de travail dans un certain nombre d'industries d'Europe; d'indiquer la nature des principaux programmes sociaux organisés par les employeurs et particulièrement ceux qui sont financés en tout ou en partie par les employeurs; de montrer l'importance relative des apports fournis dans ces programmes par les employeurs, les travailleurs et l'Etat; et enfin de jeter incidemment de la lumière sur certains éléments de revenu du travailleur en dehors du salaire en autant que ceuxci sont financés par les employeurs. Dans cette étude, on évalue plutôt le coût du travail que le revenu du travailleur. Certains éléments du coût de la main d'oeuvre assumés par l'employeur, comme, par exemple le recrutement ne peuvent pas être considé- 
rés en aucune façon comme un revenu pour les travailleurs. Tandis que d'autres, comme les contributions pour un plan de pension, vont bénéficier aux travailleurs qui ne sont plus à l'emploi de l'entreprise. De plus, ce n'est que par accident que les auteurs ont touché à ces bénéfices que les travailleurs retirent soit du gouvernement ou de leurs propres contributions.

L'enquête a porté sur les industries suivantes: textile cotton, chaussure, radio, outillage, chantiers maritimes, acier, mines de charbon et chemins de fer. Et les pays couverts sont l'Autriche, la Belgique, le Danemark, la France, l'Allemagne de l'Ouest, la Grèce, l'Italie, la Turquie, le Royaume Uni et la You. goslavie.

L'ouvrage est divisé en quatre chapîtres. La procédure suivie dans l'enquête; les grandes catégories des items qui entrent dans le coût du travail selon les établissements; les items individuels du coût du travail selon les établissements, l'importance des salaires et des suppléments de salaire dans l'ensemble de l'économie.

On ne saurait trop souligner l'utilité d'études semblables.

G. D.

A History of American Labor, by Joseph G. Rayback, The MacMillan Company, New York 1959, BrettMacMillan Ltd., 132 Water Street South Galt, Ont. $\$ 6.00$

Voici un autre ouvrage qui s'ajoute à la littérature sur le mouvement ouvrier américain. En moins de cinq cents pages, l'auteur retrace les événements qui ont marqué la naissance, le développement, les luttes des syndicats ouvriers depuis la période coloniale jusqu'à l'enquête McClellan, et il essaie de les présenter dans leur contexte économique et politique.

Bien que l'auteur nous donne à la fin de son volume une bibliographie des principaux ouvrages dont il s'est servi, l'absence de notes et référenoes dans son texte nous empêche de contrôler ses sources et de vérifier ses allégations. $\mathrm{La}$ lecture en est rendue plus facile, mais elle ne peut satisfaire ceux qui recherchent la rigueur scientifique.

Ainsi, on aurait aimé pouvoir vérifier certaines affirmations qui ne cadrent point avec ce que l'on a l'habitude de soutenir. Par exemple, à la page 155 , d'un mot, l'auteur nous dit que Gompers n'a jamais été un socialiste.

Dans l'ensemble, c'est plutôt un ouvrage superficiel. Mais des lecteurs qui n'ont pas beaucoup de temps à disposer et qui veulent pourtant se familiariser avec l'histoire du syndicalisme aux Etats-Unis trouveront dans l'étude du Dr Rayback le livre qu'il leur faut.

$$
\text { G. D. }
$$

Conflict within the AFL, a study of craft versus industrial unionism, 19011938. By James O. Morris. Cornell University, Ithaca, New York, 1958. 319 pp. $\$ 5.00$

La scission qui s'est produite au sein de l'AFL par la création du CIO a été l'aboutissement d'une longue suite de divergences qui n'ont pu trouver de voies de compromis à l'intérieur de la grande centrale américaine et qui s'échelonnent de 1901 à 1935.

Etudier de près l'histoire de ces divergences, les analyser sous tous leurs angles est nécessaire si l'on veut connaître la dynamique qui caractérise le mouvement ouvrier américain. Non pas que l'histoire du mouvement ouvrier aux Etats-Unis se confonde avec celle de l'AFL, car même pendant cette période, à côté de cette centrale il a toujours existé d'autres groupements indépendants. Mais parce que l'on trouve au sein de l'AFL à la fois une tentative de grouper démocratiquement diverses tendances et aussi un échec.

Dans cet ouvrage, ainsi que l'indique le titre, l'auteur se borne à décrire et à interpréter le conflit qui a régné dans l'AFL pendant une période de trente ans. Ce conflit, s'il est surtout apparu comme celui d'une lutte entre deux formules d'organisations, les unions de métiers et les unions industrielles, était beaucoup plus profond. Au fond, c'était un conflit idéologique qui impliquait aussi une conception de l'or- 